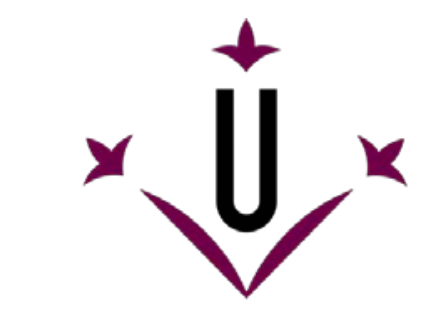

Universitat de Lleida

Document downloaded from:

http://hdl.handle.net/10459.1/65125

The final publication is available at:

https://doi.org/10.1021/acs.analchem.5b00606

Copyright

(c) American Chemical Society, 2015 


\title{
Determination of the free metal ion concentration using AGNES implemented with environmentally-friendly bismuth film electrodes
}

Luciana S. Rocha ${ }^{\mathrm{a}, *}$, Josep Galceran ${ }^{\mathrm{b}}$, Jaume Puy ${ }^{\mathrm{b}}$ \& José Paulo Pinheiro ${ }^{\mathrm{c}}$

${ }^{a}$ CIQA, DQF/FCT, University of Algarve, 8005-139 Faro, Portugal.

${ }^{\mathrm{b}}$ Department of Chemistry, University of Lleida and AGROTECNIO, Rovira Roure 191, 25198 Lleida, Spain

c Laboratoire Interdisciplinaire des Environnements Continentaux, UMR 7360 CNRS Université de Lorraine, 15 avenue du Charmois, 54500 Vandoeuvre-les-Nancy, France

\begin{abstract}
Ex-situ plated Bi film electrodes (Bi-FE) have been employed, for the first time, to measure the free concentration of $\mathrm{Pb}(\mathrm{II})$ in aqueous solutions using AGNES (Absence of Gradients and Nernstian Equilibrium Stripping) with Stripping Chronopotentiometry (SCP) quantification. The amount of deposited $\mathrm{Pb}^{\circ}$, below a certain threshold, follows a Nernstian relationship with the applied potential. This threshold can be interpreted as the frontier of transition from surface deposition to solid (bulk) formation of $\mathrm{Pb}^{\circ}$. AGNES

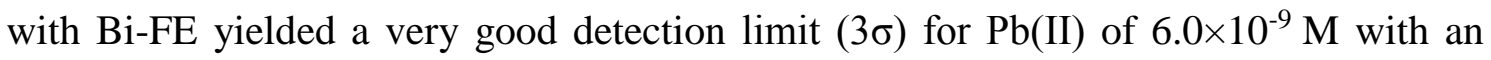
applied gain of 398 and a deposition time of $400 \mathrm{~s}$. The ability of the Bi film electrode to perform speciation measurements was demonstrated for $\mathrm{Pb}(\mathrm{II})-\mathrm{PSS}$ and $\mathrm{Pb}(\mathrm{II})-\mathrm{IDA}$ systems. The measured values with the Bi-FE were in good agreement with the values obtained using the Hg film electrode and/or the values reported in the literature.
\end{abstract}

Keywords: AGNES; Bi film electrode; Lead; Stability constants; Speciation *Corresponding author. E-mail: lsrocha@ualg.pt. Tel: +351 289800900. 


\section{INTRODUCTION}

The knowledge of the free ion concentration is fundamental to predict the bio-uptake of trace metals in natural aquatic systems ${ }^{1 ; 2}$. A limited number of analytical techniques including Donnan Membrane Tecnique (DMT) $3 ; 4$, Complexing Gel Integrated MicroElectrode $^{5}$ (CGIME), Permeation Liquid Membrane (PLM) ${ }^{6 ; 7}$ or Potentiometry with Ion Selective Electrodes (ISE) ${ }^{8}$ can be employed for the determination of the free metal ion concentrations in natural systems. However, ISE generally suffers from lack of sensitivity and the interference from other metal ions, and the commercial Pb-ISE requires total $\mathrm{Pb}(\mathrm{II})$ above micromolar ${ }^{9}$.

In the last decade, Absence of Gradients and Nernstian Equilibrium Stripping (AGNES) has been specifically designed ${ }^{10}$ and developed to determine the free metal ion concentration in aquatic systems ${ }^{11-14}$, overcoming some of the limitations of the former methods. This electroanalytical method consists of two conceptual stages: the deposition and stripping stages. Along the first stage, the metal ion in solution $\mathrm{M}^{2+}$ is reduced to $\mathrm{M}^{0}$, until a situation of Nernstian equilibrium with absence of gradients in the concentration profiles is attained. The aim of the second stage ${ }^{10}$, quantification of the accumulated $\mathrm{M}^{\mathrm{o}}$, can be achieved via different strategies leading to different variants ${ }^{15}$. In this work, Stripping Chronopotentiometry (SCP), which allows the discrimination of interferences and avoids blank measurements, is selected as the second stage of AGNES. A constant oxidizing stripping current is applied until full depletion ${ }^{16}$.

Typically, AGNES has been implemented with mercury (Hg) electrodes, including the Hanging Mercury Drop Electrode (HMDE) ${ }^{17}$, the Hg-Ir microelectrode ${ }^{18}$, Thin Mercury Film Elctrode (TMFE) ${ }^{19}$ and the Hg Screen Printed Electrode (SPE) ${ }^{16 ; 20-22}$. While these small-volume Hg electrodes offer an attractive performance in the stripping analysis of trace metal ions, alternative electrode materials, with a similar performance, 
are urgently needed for addressing growing concerns regarding the toxicity, volatility and disposal of this element. Bismuth is one of the materials that shows attractive electrochemical features and Bi film electrodes (Bi-FE) have been extensively used in the stripping analysis of trace metals ${ }^{23-26}$. Recently, the bismuth film electrode was successfully used for speciation analysis of trace metals by stripping chronopotentiometry (SCP) ${ }^{27 ; 28}$. With the growing demand for on-site analysis, the use of non-toxic Bi-FE can represent a step ahead in going from laboratory to field studies.

The aim of the present work is to describe the implementation of AGNES with Bi-FE electrodes. To achieve this goal, the specific characteristics of ex-situ Bi-FE and the required experimental conditions of AGNES technique in the determination of the free concentration of lead(II) will be explored. The metal speciation capability of AGNES-SCP will be tested with polystyrene sulfonate (PSS) (labile system) and iminodiacetate (IDA) (non-labile system). The results will be compared with the data obtained using $\mathrm{Hg}$ film electrodes and with the existing theory for quantitative interpretation of SSCP curves.

\section{MATERIALS and METHODS}

\section{Reagents}

The chemicals used in the present work were of analytical reagent grade and used asreceived, unless stated otherwise. All solutions were prepared with ultra-pure water (18.3 $\mathrm{M} \Omega \mathrm{cm}$, Milli-Q systems, Millipore-waters). Nitric acid 65\% (suprapur) and the standard stock solutions of mercury nitrate $\left(1001 \pm 2 \mathrm{mg} \mathrm{L}^{-1}\right)$ and lead nitrate $\left(999 \pm 2 \mathrm{mg} \mathrm{L}^{-1}\right)$ were purchased from Merck. Pb(II) solutions were prepared from dilution of the certified standard. Sodium nitrate electrolyte solution (0.01M) and MES (2-(N-morpholino) 
ethanesulfonic acid) buffer (0.2 M) were prepared from the solids (Merck, suprapur and Merck >99\%, respectively). Solutions prepared from nitric acid (Merck, suprapur) and sodium hydroxide (0.1 M standard, Merck) were used to adjust the $\mathrm{pH}$. Potassium thiocyanide, hydrochloric acid, potassium chloride were all p.a. from Merck and were used to prepare the solution for the re-dissolution of the mercury film. Potassium ferricyanide (Merck p.a.) was used to prepare a standard solution $\left(1.124 \times 10^{-3} \mathrm{M}\right)$ in 1.0 M potassium chloride for the determination of the carbon electrode area. Solutions of ammonium acetate $\left(\mathrm{NH}_{4} \mathrm{CH}_{3} \mathrm{COO}(1.0 \mathrm{M}) / \mathrm{CH}_{3} \mathrm{COOH}(1.0 \mathrm{M})\right)$ (Merck) were prepared monthly and used without further purification. Sodium styrenesulfonate, S(Thiobenzoyl)-thioglycolic acid, 4,4'-Azobis(4-cyanovaleric acid) and toluene from Sigma-Aldrich and methanol from Panreac were used in the synthesis of $4000 \mathrm{Da}$ poly(styrenesulfonate).

\section{Synthesis of monodisperse charged 4000 Da (PSS) $)_{n}-\mathrm{COOH}$ polymer}

Samples of poly(styrenesulfonate) of 4,10 , and $30 \mathrm{kDa}$ were obtained by reversible addition-fragmentation chain transfer (RAFT) polymerization of sodium styrenesulfonate in a water/ethanol solution, using S-(Thiobenzoyl)-thioglycolic acid as chain transfer agent (CTA) and 4,4'-Azobis(4-cyanovaleric acid) as radical initiator ${ }^{29}$. Further details regarding the synthesis of $4000 \mathrm{Da}(\mathrm{PSS})_{\mathrm{n}}-\mathrm{COOH}$ are described elsewhere ${ }^{30}$.

\section{Apparatus}

An Ecochemie Autolab PGSTAT12 potentiostat (controlled by GPES 4.9 software from EcoChemie, the Netherlands) was used in conjunction with a Methrohm 663 VA stand (Methrohm, Switzerland). The three electrode configuration consisted of a Bi film plated onto a rotating glassy carbon (GC) disk (2 mm diameter, Metrohm) as the working electrode, a GC rod counter electrode and an Ag/AgCl reference electrode from 
World Precision Instruments, DRIREF-5 (electrolyte leakage $<8 \times 10^{-4} \mu \mathrm{L} / \mathrm{h}$ ). A Denver Instrument (model 15) and a Radiometer analytical combination $\mathrm{pH}$ electrode, calibrated with Titrisol buffers (Merck), were used to measure $\mathrm{pH}$.

\section{Preparation of the glassy carbon substrate}

Prior to deposition of the Bi films, the GC electrode was conditioned following a previously reported polishing/cleaning procedure ${ }^{31}$. In brief, the electrode was polished with alumina slurry (grain size $0.3 \mu \mathrm{m}$, Metrohm) and sonicated in pure water for $60 \mathrm{~s}$, to obtain a renewed surface. Then, an electrochemical pre-treatment was carried out using 50 cyclic voltammetric scans between -0.800 and $+0.800 \mathrm{~V}$ at $0.1 \mathrm{~V} \mathrm{~s}^{-1}$, in $\mathrm{NH}_{4} \mathrm{CH}_{3} \mathrm{COO}$ $(1 \mathrm{M}) / \mathrm{HCl}(0.5 \mathrm{M})$ solution. The area of the glassy carbon electrode (geometrical value of $3.14 \mathrm{~mm}^{2}$ ) was measured by chronoamperometry in $1.124 \times 10^{-3} \mathrm{M}$ ferricyanide/1.0 $\mathrm{M}$ $\mathrm{KCl}$ solution (purged with $\mathrm{N}_{2}$ for 300 s). Before the chronoamperometric measurements, the solution was stirred for $30 \mathrm{~s}$ (2000 rpm) followed by a resting period of $120 \mathrm{~s}$. The chronoamperometric parameters used were: $E=0.500 \mathrm{~V}$ and $t=3 \mathrm{~s}$. The electrochemically active area of the GC electrode was calculated from the slope of $I$ vs $t^{-1 / 2}$ Cottrell equation (diffusion coefficient of ferricyanide $D=7.63 \times 10^{-10} \mathrm{~m}^{2} \mathrm{~s}^{-1}$ ) and the value obtained was $3.334 \pm 0.062 \mathrm{~mm}^{2}$ (two polishing experiments, each with four replicate determinations). When not in use, the bare GC electrode was stored dry in a clean atmosphere.

\section{Preparation of the Bismuth film electrode}

The ex-situ Bi film was prepared adopting the procedure described by Kong et al. ${ }^{32}$, by electrodeposition at $-0.300 \mathrm{~V}$ for 120 s at a rotation speed of $1000 \mathrm{rpm}$ and using a solution containing both $8 \times 10^{-4} \mathrm{M}$ of $\mathrm{Bi}^{3+}$ and $8 \times 10^{-4} \mathrm{M}$ of $\mathrm{Sn}^{2+}$ prepared in $\mathrm{KCl}(1 \mathrm{M})$ and $\mathrm{HCl}(0.13 \mathrm{M})$ media (pH ca. 0.74). According to those authors, Sn(II) ions in the 
electrolyte block the migration of $\mathrm{Bi}(\mathrm{III})$ ions during the deposition, whilst $\mathrm{Sn}(\mathrm{II})$ ions cannot be deposited at the applied potential $(-0.3 \mathrm{~V}$, which was more negative than $E_{\mathrm{Bi}(\mathrm{III}) / \mathrm{Bi}}$, but more positive than $E_{\mathrm{Sn}(\mathrm{III} / \mathrm{Sn})}$. As a result, the bismuth film obtained showed a compact crystal layer, a high coverage ratio and tightly and homogeneously distributed Bi deposits on the GC surface. The charge associated with the deposited Bi $\left(Q_{\mathrm{Bi}}=1.65 \times 10^{-3} \mathrm{C}\right)$ was calculated by electronic integration of the linear sweep stripping peak of $\mathrm{Bi}$, for $v=0.005 \mathrm{~V} \mathrm{~s}^{-1}$. The electrolyte solution was $\mathrm{H}_{2} \mathrm{SO}_{4} 0.5 \mathrm{M}$ and the stripping step began at $-0.150 \mathrm{~V}$ and ended at $+0.400 \mathrm{~V}^{27}$. The thickness estimated for the Bi film electrode was 73.6 $\pm 0.6 \mathrm{~nm}$ (mean value of 3 replicate measurements).

\section{AGNES measurements: optimization studies}

Measurements were carried out in $20 \mathrm{~mL}$ of 0.01 and $0.1 \mathrm{M} \mathrm{NaNO}$ solutions containing $\mathrm{Pb}(\mathrm{II})$ concentrations close to $10^{-7} \mathrm{M}$ at room temperature $\left(21-23^{\circ} \mathrm{C}\right)$. All solutions were purged for 15 min with $\mathrm{N}_{2}$ at the beginning of every experiment and for 20 s (with mechanical stirring of the rotating disk electrode, RDE) after each measurement. The free metal ion concentration was determined with the variant AGNES$\mathrm{SCP}^{16}$. In stage 1 , at a fixed deposition potential $E_{1}$, mass transfer was enhanced by rotation of the RDE at $1000 \mathrm{rpm}$. In stage 2, a stripping oxidising current $I_{\mathrm{s}}$ of $0.5 \mu \mathrm{A}$ was applied until the potential reached $-0.300 \mathrm{~V}$.

Prior to each AGNES-SCP experiment, the peak potential ( $\left.E_{\text {peak,DPV }}\right)$ value of lead(II) in the solution used in the experiments was determined by differential pulse voltammetry (DPV). The deposition step lasted $30 \mathrm{~s}$ at $-0.200 \mathrm{~V}$, while the solution was stirred, followed by a resting period of $5 \mathrm{~s}$. The stripping step was initiated at $-0.320 \mathrm{~V}$ and ended at $-0.70 \mathrm{~V}$; the DPV parameters used were: amplitude $0.025 \mathrm{mV}$ and step potential $0.002 \mathrm{~V}$. 


\section{Theoretical framework for the application of AGNES with solid electrodes}

A key requirement for AGNES is the attainment of Nernstian equilibrium by the end of the first stage (i.e. after a sufficiently long deposition time $t_{1}$ ). For a deposition potential $E_{1}$, equilibrium means:

$$
E_{1}=E^{0}+\frac{R T}{n F} \ln \frac{\left\{\mathrm{M}^{n+}\right\}}{\left\{\mathrm{M}^{0}\right\}}
$$

where $E^{0}$ is the standard electrode potential, $R$ the gas constant, $T$ the temperatue, $n$ the number of exchanged electrons, $F$ the Faraday constant and curly brackets indicate activity of the enclosed species ( $\mathrm{M}^{n+}$ for the analyte; $\mathrm{M}^{0}$ for its reduced couple).

In mercury electrodes (the only ones reported up now for AGNES since its introduction in 2004), an amalgamating element ( $\mathrm{Zn}, \mathrm{Cd}, \mathrm{Pb})$ is accumulated until equilibrium. Nernst law prescribes the eventual relationship between the activities of the free metal ion in solution and of the reduced metal in the amalgam (see eqn. (1)). So, by changing the deposition potential, one changes the ratio between activities (which, for analytical purposes, is converted into a ratio of concentrations called pre-concentration factor or gain, $Y)^{10}$.

If one uses a solid electrode and $\mathrm{M}^{\mathrm{o}}$ deposits on it as a bulk solid phase, its activity is always unity, regardless how much metal has been deposited. So, one would switch from no deposition at all, for more positive potentials, to unlimited deposition (never reaching of equilibrium) for more negative potentials ${ }^{33}$, which could be labelled "runaway deposition”. Thus, bulk solid deposition, the normal process on solid electrodes, seems not compatible with AGNES. 
However, the possibility of a varying activity of $\mathrm{M}^{\mathrm{o}}$ with solid electrodes is known in electrochemisty: the activity is related to the coverage of adsorbed atoms on the solid surface (e.g. below the complete formation of a monolayer):

$\left\{\mathrm{M}^{0}\right\}=\gamma_{\mathrm{M}^{0}} \theta$

where $\gamma_{\mathrm{M}^{0}}$ is the activity coefficient and $\theta$ is the coverage (ratio between the current surface concentration $\Gamma_{\mathrm{M}^{0}}$ and the maximum one $\Gamma_{\mathrm{M}^{0} \text {,max }}$ which is also the ratio between the current number of adsorbed moles $n_{\mathrm{M}^{0}}$ and the maximum one):

$\theta=\frac{\Gamma_{\mathrm{M}^{0}}}{\Gamma_{\mathrm{M}^{0}, \max }}=\frac{n_{\mathrm{M}^{0}}}{n_{\mathrm{M}^{0}, \max }}$

For this kind of system, assuming $\gamma_{\mathrm{M}^{0}}=1$, Nernst law is often re-casted as ${ }^{34}$ :

$E_{1}=E^{0}+\frac{R T}{n F} \ln \frac{\left\{\mathrm{M}^{n+}\right\}}{\theta}$

So, we define the gain as

$Y \equiv \frac{\theta}{\left[\mathrm{M}^{n+}\right] / C^{0}}$

where $c^{0}$ is the standard state concentration of $1 \mathrm{~mol} \mathrm{~L}^{-1}$ (a subscript $\theta$ could be added to $Y$ as a reminder of the coverage implication, but for the sake of simplicity in this work this subscript is spared).

The aim of the second stage is quantification of the amount of $\mathrm{M}^{\mathrm{o}}$ once equilibrium is attained. In the variant that uses stripping chronopotentiometry (SCP) in the second stage, the time for complete depletion (transition time, $\tau$ ) can be determined from the evolution 
of the recorded potential in response to the imposed stripping current $I_{\mathrm{s}}{ }^{21}$. The faradaic charge $(Q)$ can be rigorously computed ${ }^{16}$ as:

$Q=\left(I_{\mathrm{S}}-I_{\mathrm{Ox}}\right) \tau$

where $I_{\mathrm{ox}}$ is the current due to other oxidants. Depending on the $I_{\mathrm{s}}$ value applied, the contribution from the current due to the presence of other oxidants $\left(I_{O x}\right)$ can be negligible when compared with the stripping current $\left(I_{\mathrm{s}}>>I_{\mathrm{Ox}}\right)$ and the charge $Q$ (rigorously computed with eqn. (6)) can be simplified to:

$Q=I_{S} \tau$

Under the experimental conditions used to perform AGNES measurements at Bi film electrodes, i.e., for the $I_{\mathrm{S}}$ values of 0.5 and $1 \mu \mathrm{A}$, the charge $Q$ was determined using eqn. (7) (for further details see section S1 of the supporting information).

On the other hand, with Faraday law, this charge can be computed from the number of adsorbed moles that are converted from $\mathrm{M}^{\mathrm{o}}$ to $\mathrm{M}^{\mathrm{n}}$ in the stripping stage, so:

$Q=n F n_{\mathrm{M}^{0}}=n F A \Gamma_{\mathrm{M}^{0}, \max } \theta=\left(\frac{n F A \Gamma_{\mathrm{M}^{0}, \max }}{c^{0}}\right) Y\left[\mathrm{M}^{n+}\right]$

where $A$ is the active area for adsorption.

By gathering the terms inside the brackets, the direct proportionality between the analytical signal and the free concentration of analyte is evidenced:

$Q=\eta_{\theta} Y\left[\mathrm{M}^{n+}\right]$

where $\eta_{\theta}$ is a proportionality factor characteristic of the number of exchanged electrons and an extensive property of the solid electrode (parallel to the previously used $\eta_{\mathrm{Q}}$ which 
was characteristic of the volume of mercury electrodes $)^{15 ; 35}$. The product $Y \eta_{\theta}$ can be experimentally found from a calibration.

The gain can be controlled through the applied potential $\left(E_{1}\right)$, whose value could be computed using a rigorous expression in terms of $E^{\mathrm{o}}$ (just by re-arrangement of eqn. (4)). In practice, the experimental determination of an accurate value of $E^{0}$ might be involved, so, in this work, we just use the potential peak of a Differential Pulse Voltammogram as a reference potential for the computation of the gain. Fortunately, the offset (between the exact gain and its surrogate) that might appear in the calibration completely cancels out with the offset in the measurement, so that a perfect knowledge of the gain is not essential for analytical purposes (i.e. AGNES quantification essentially relies in a calibrated proportionality between $Q$ and free concentration, see eqn. (9)). Thus, here, we operationally report the gains with the expression:

$$
Y=\exp \left[-\frac{n F}{R T}\left(E_{1}-E_{\text {peak,DPV }}\right)\right]
$$

This means that the strength of the adsorption is controlled by the applied potential $\left(E_{1}\right)$.

\section{RESULTS and DISCUSSION}

\section{Implementation of AGNES-SCP with Bi film electrode}

The following preliminary studies were performed: i) the effect of the deposition potential $E_{1}$ and the deposition time $t_{1}$ required to reach AGNES conditions and ii) the stability of the electrode along several consecutive AGNES measurements. 


\section{Trajectories $Q$ vs $t_{1}$ at different $E_{1}$}

In this section it is demonstrated that Nernstian equilibrium is effectively reached for certain deposition potentials $E_{1}$ for sufficiently long deposition times $t_{1}$. Figure 1 shows a collage of panels each one with the trajectory (i.e. plot of the analytical response of a set of AGNES experiments as a function of increasing deposition times) for different deposition potentials $E_{1}$. The $E_{1}$ values used to perform these experiments were selected according with a preliminary experiment using Scanning Stripping ChronoPotentiometry (SSCP), i.e. $E_{1}$ did not exceed the $E_{\mathrm{d}, 1 / 2}$ of the SSCP curve (for further details see section S2 of the Supporting Information).
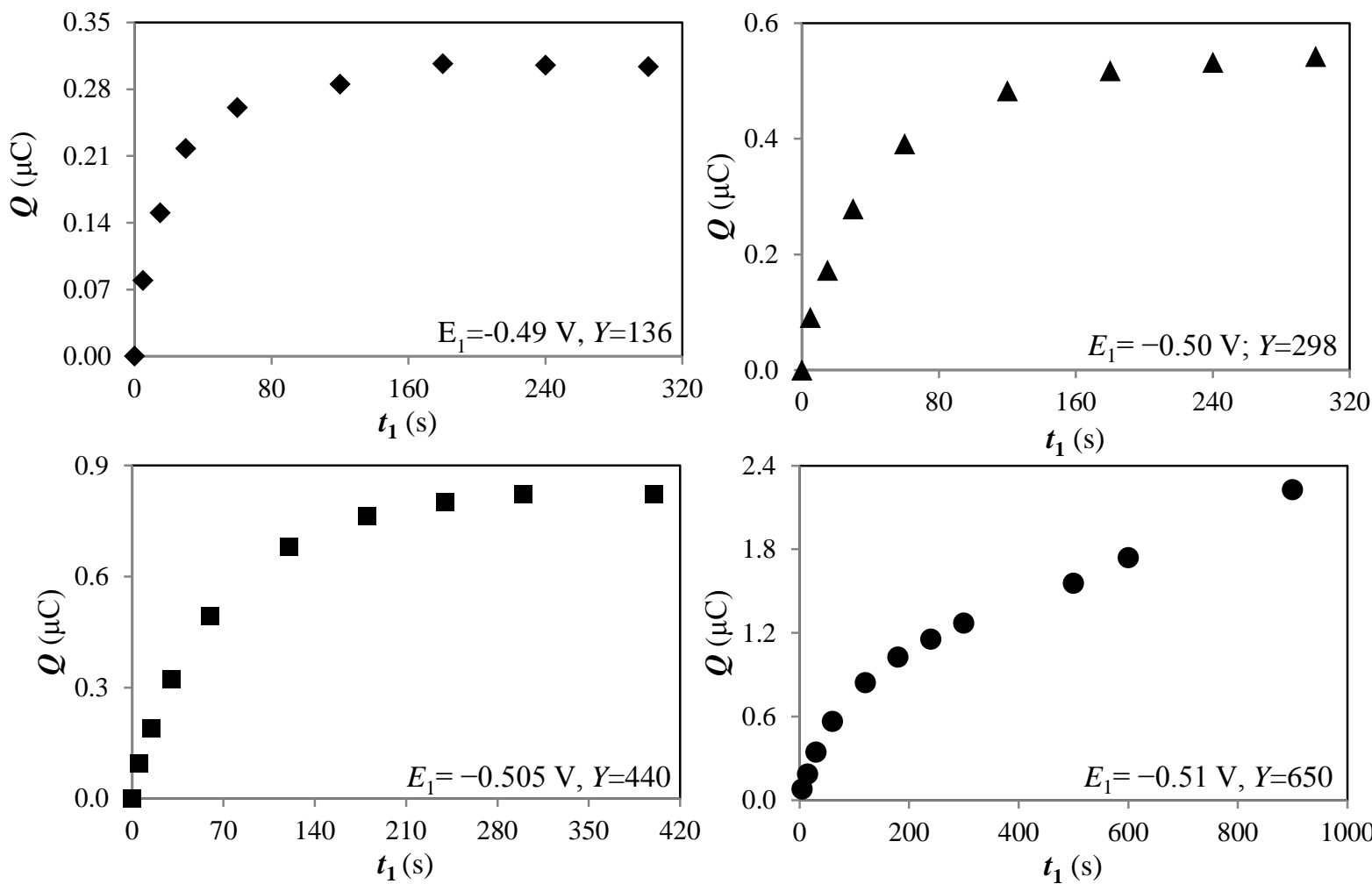

Figure 1. Trajectories ( $Q$ vs $t_{1}$ ) obtained in $\mathrm{NaNO}_{3} \quad 0.01 \mathrm{M}$ and for $\left[\mathrm{Pb}^{2+}\right]$ of $4.6 \times 10^{-7} \mathrm{M}$ $\left(c_{\mathrm{T}, \mathrm{Pb}}=5.0 \times 10^{-7} \mathrm{M}\right)$ using the following deposition potentials:-0.490 V $(\triangleleft),-0.500 \mathrm{~V}(\boldsymbol{\Delta}),-0.505$ $\mathrm{V}(\boldsymbol{\bullet})$ and $-0.510 \mathrm{~V}(\bullet)$. Other experimental conditions: $I_{\mathrm{s}}=5 \times 10^{-7} \mathrm{~A}, E_{\text {limit }}=E_{\text {standby }}=-0.300 \mathrm{~V}$, $t_{\text {max,meas }}=20 \mathrm{~s}, v_{\mathrm{RDE}}=1000 \mathrm{rpm}$. 
The stabilization of the charges in Figure 1 for most of the deposition potentials from a certain deposition time on is a clear indication of an equilibrium being reached. Experimental evidence is consistent with ascribing this equilibrium to the adsorption of reduced atoms (probably forming a monolayer) in the line of the theory expound in section 3. The time required to reach this equilibrium $\left(t_{1}\right)$ depends on the deposition potential $E_{1}$, i.e., on the gain $Y$ selected. The results show that higher gains require longer deposition times $t_{1}$ and that the equilibrium (identified as a plateau in the $Q=f\left(t_{1}\right)$ graph) is attained for a deposition time $t_{1}$ of $180 \mathrm{~s}$ for a $E_{1}$ of $-0.490,240 \mathrm{~s}$ for $-0.500 \mathrm{~V}$ and 300 $\mathrm{s}$ for $E_{1}$ of $-0.505 \mathrm{~V}$. The trajectory for $E_{1}=-0.510 \mathrm{~V}$ in figure 1 does not reach equilibrium in the probed deposition times. The shape of the trajectory can speculatively be interpreted as follows: above a certain threshold of the product $\mathrm{Pb}(\mathrm{II})$ concentration times the gain (which is a certain coverage as indicated by eqn. (5)), the deposited lead is no longer restricted to a monolayer and bulk lead (runaway) deposition starts. In this situation, Nernst equilibrium is no longer possible due to the unity activity of the solidphase deposited lead. Whatever the physical phenomena involved, the practical consequence is an upper limit of linearity between free metal concentration and the measured charge. A similar restriction also applies to mercury electrodes (when there is saturation of the amalgam) $)^{35}$. This kind of limitations can be overcome by using lower gains.

\section{Stability of Bi film electrodes}

The stability and repeatability of Bi film electrode prepared following Kong et $a l .{ }^{32}$ was assessed by performing several consecutive AGNES measurements (Figure 2). 


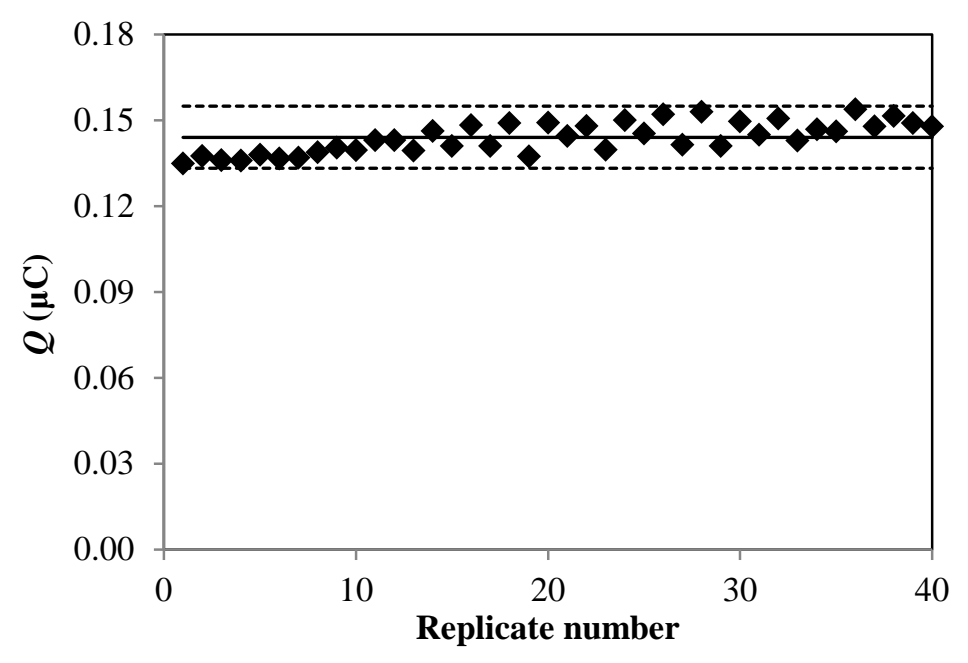

Fig.2. AGNES-SCP signal (Q) for repeated measurements of $\mathrm{Pb}(\mathrm{II})$ at Bi film electrode ( $\bullet)$, where the lines represent: (-) $Q$ average value and (---) the standard deviation $(2 \sigma)$. The experiments were performed in $0.01 \mathrm{M} \mathrm{NaNO}$ for $\left[\mathrm{Pb}^{2+}\right]=2.8 \times 10^{-7} \mathrm{M}\left(c_{\mathrm{T}, \mathrm{Pb}}=3.0 \times 10^{-7} \mathrm{M}\right)$ with the following parameters: $E_{1}=-0.495 \mathrm{~V}, t_{1}=300$ s. Other conditions as in Figure 1.

There was a slight increase in the response charge (ca. 14\%) along the 40 consecutive measurements, the same tendency already reported previously by Rocha et al. ${ }^{27}$, when using the ex-situ plated Bi film electrode to measure Pb(II) by SCP. Nevertheless the overall relative standard deviation was just $7.5 \%(2 \sigma)$, showing that this electrode can be used along a considerable set of measurements, with no relevant change in the active surface area.

\section{Calibrations of $\mathrm{Pb}(\mathrm{II})$ with Bi film electrodes}

Calibration plots were obtained at different deposition potentials in $\mathrm{NaNO}_{3} 0.01 \mathrm{M}$ media and at $\mathrm{pH}$ ca. 4. The potentials selected to perform these experiments were: -0.485 , -0.495 and $-0.505 \mathrm{~V}$, that correspond to gains $Y$ of 51, 182 and 398, respectively. In order to ensure AGNES conditions, the measurements were performed until consistency using different deposition times. The selection of $t_{1}$ values was highly dependent on the $E_{1}$ applied and it was based on $Q$ vs $t_{1}$ trajectories obtained in section 4.1.1. Table 
presents the calibration data: slope and correlation coefficient and the limit of detection (LOD).

Table 1. Calibration data: slope $(m)$ and correlation coefficient $(r)$ of the $Q v s$. [ $\left.\mathrm{Pb}^{2+}\right]$ calibration plots and the limit of detection (LOD).

\begin{tabular}{ccccc}
$\boldsymbol{Y}$ & $\boldsymbol{m} \pm \mathrm{Sm}\left(\mathrm{C} \mathrm{M}^{-1}\right)$ & $\boldsymbol{r}$ & $\boldsymbol{n}$ & $\mathrm{LOD}(\mathrm{M})$ \\
\hline 51 & $(3.43 \pm 0.06) \times 10^{5}$ & $0.999_{(4)}$ & 6 & $3.42 \times 10^{-8}$ \\
182 & $(1.11 \pm 0.01) \times 10^{6}$ & $0.999_{(9)}$ & 7 & $1.14 \times 10^{-8}$ \\
398 & $(2.26 \pm 0.02) \times 10^{6}$ & $0.999_{(9)}$ & 5 & $5.99 \times 10^{-9}$ \\
\hline
\end{tabular}

The measured charge increases linearly with the free concentration of $\mathrm{Pb}(\mathrm{II})$, however the interval of linearity is highly dependent on the deposition potential $E_{1}$, i.e. on the gain $Y$ applied. For some deposition potential $E_{1}$, there is a deviation from the linearity resulting from "runaway deposition” whose detailed analysis will be provided in section 4.3.

The limits of detection (LOD) of AGNES-SCP at Bi film electrode were: $3.4 \times 10^{-8}$ $\mathrm{M}$ ( $n=6$ and $\left.r=0.999_{4}\right), 1.14 \times 10^{-8} \mathrm{M}(n=7$ and $r=0.9999)$ and $6.0 \times 10^{-9} \mathrm{M}(n=5$ and $r=0.9999)$, for $Y$ of $51\left(t_{1}=200 \mathrm{~s}\right), 182\left(t_{1}=300 \mathrm{~s}\right)$ and $398\left(t_{1}=400 \mathrm{~s}\right)$ respectively. Lower LOD can be achieved by applying higher gains $Y$, however longer deposition times $t_{1}$ are required to attain AGNES conditions. As previous slopes represent the product $Y \eta_{\theta}$, one can recover an average $\eta_{\theta}=(6.17 \pm 0.52) \times 10^{3} \mathrm{C} \mathrm{M}^{-1}$ with the computation of $Y$ given by eqn. (10). The practical constancy of $\eta_{\theta}$ is indicative of the Nernstian behaviour (i.e. according to eqn. (9) and (10)) of AGNES signals. This Nernstian behaviour is also confirmed by the linearity of the plots $Q$ vs. $Y$ (see Fig S3 in the supporting information). 


\section{A theoretical interpretation of the results}

According to Herzog and Arrigan ${ }^{36}$, the metal deposition in solid electrodes can, in some systems, occur in two distinct steps: 1) the underpotential deposition (UPD) that is restricted to the formation of a monolayer of reduced metal, and 2) the bulk metal deposition leading to the formation of several layers of metal atoms on the electrode surface. UPD of $\mathrm{Pb}$ has been intensively investigated on gold ${ }^{37-40}$ and silver ${ }^{41 ; 42}$ solid electrodes, but no studies in the literature reported this phenomenon on Bi electrodes. Szabo provided a detailed theoretical treatment of UPD ${ }^{43}$.

Results in this work can be tentatively interpreted assuming a key role to adsorption of $\mathrm{Pb}^{\circ}$ modulated by the applied deposition potential:

$$
\text { (free adsorption sites) }+\mathrm{Pb}^{2+}+2 \mathrm{e}^{-} \stackrel{K^{\text {cond }}}{\rightleftarrows}\left(\mathrm{Pb}^{0}\right) \text { ads }
$$

The conditional equilibrium constant $K^{\mathrm{cond}}$, which depends on the applied potential, involves concentrations in solution and in the adsorbed surface (i.e. activity coefficients, assumed to be constant, are embedded in it):

$$
K^{\mathrm{cond}}=\frac{\Gamma_{\mathrm{Pb}^{0}}}{\left(\Gamma_{\mathrm{Pb}^{0}, \text { max }}-\Gamma_{\mathrm{Pb}^{0}}\right)\left[\mathrm{Pb}^{2+}\right]}
$$

and re-organized as:

$$
\theta=\frac{\Gamma_{\mathrm{Pb}^{0}}}{\Gamma_{\mathrm{Pb}^{0}, \max }}=\frac{K^{\text {cond }}\left[\mathrm{Pb}^{2+}\right]}{1+K^{\text {cond }}\left[\mathrm{Pb}^{2+}\right]}
$$

which is just a Langmuirian isotherm. This reverts to the linear (Henry) isotherm for sufficiently low coverages:

$$
\theta=K^{\text {cond }}\left[\mathrm{Pb}^{2+}\right]
$$

Comparison with equation (5), allows numerically identifying $Y$ with $K^{\text {cond }}$. 
In this work, calibration curves were also obtained applying a broader range of deposition potentials, ranging from -0.485 to $-0.510 \mathrm{~V}$, corresponding to pre-concentration factors $Y$ between 51 and 370, respectively (Figure 3). For gains above $Y=51$ and above a charge of approximately $0.6 \mu \mathrm{C}$, there is a region A (white zone), where the trajectories do not reach any equilibrium (i.e. increasingly larger charges are obtained for longer deposition times). Values indicated as open markers correspond to a deposition time that would have been sufficiently long as to reach AGNES conditions for lower $\left[\mathrm{Pb}^{2+}\right]$ and the same gain. In region $\mathrm{B}$, where $Y>51$ and $Q<0.6 \mu \mathrm{C}$ (dark grey background), the retrieved charge (indicated with solid markers) is proportional to the $\left[\mathrm{Pb}^{2+}\right]$. In region $\mathrm{C}$ (pale gray zone, $Y \leq 51$ ), the linearity of charge extends to all probed concentrations, even when $Q>0.6 \mu C$. Probably the limit established for the maximum amount of adsorbed $\mathrm{Pb}^{\circ}$ is related to the nature of the Bi film electrode, in particularly with its area. Future work should further investigate this issue.

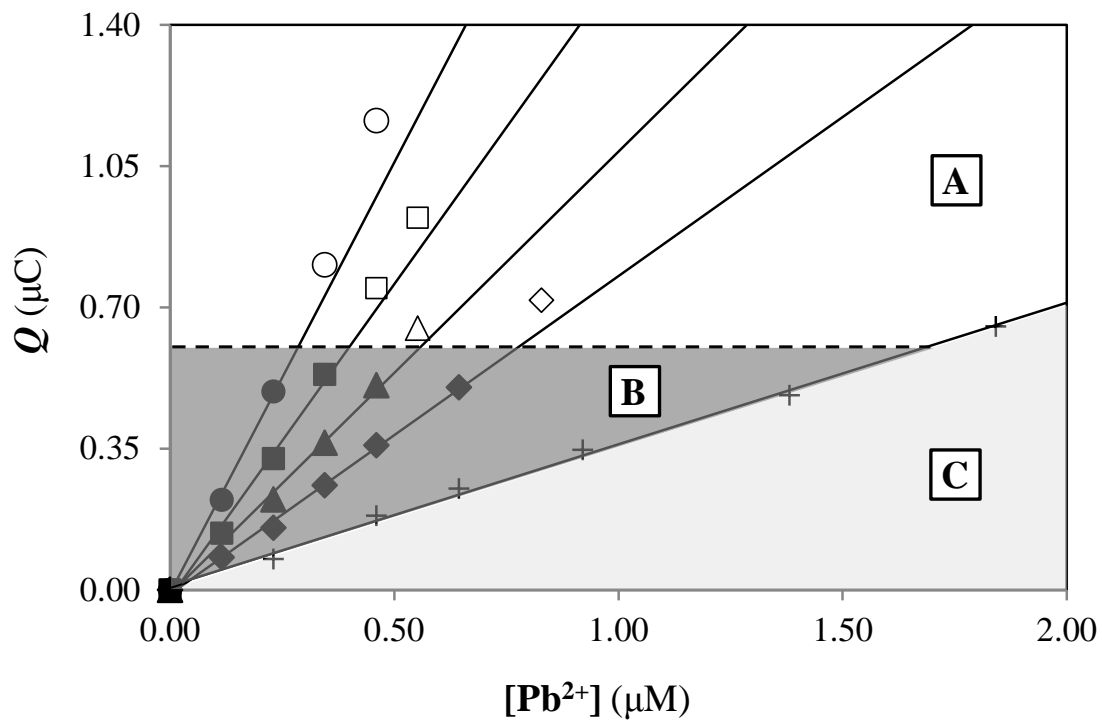

Figure 3. Variation of the charge $Q$ as a function of the free concentration of lead(II) obtained in $\mathrm{NaNO}_{3} 0.01 \mathrm{M}, \mathrm{pH} 4$ and using the following conditions: $E_{1}$ of $-0.485(+),-0.495(\diamond$ and $\diamond),-0.500$ $(\boldsymbol{\Delta}$ and $\Delta),-0.505\left(\bullet\right.$ and $\square$ ) and $-0.510\left(\bullet\right.$ and $\circ$ ) V. The deposition time $t_{1}$ used ranged between $200 \mathrm{~s}$ and $600 \mathrm{~s}$, depending on the $E_{1}$ applied. Other experimental conditions are the same as in 
Figure 1. The regions correspond to: (A) bulk deposition of $\mathrm{Pb}^{0}$, (B) linear regime below the threshold (represented by ---) and (C) linear regime even above threshold.

A possible interpretation of the regions follows:

Region A: runaway deposition (white zone). We could attribute the lack of AGNES equilibrium (i.e. open markers in Fig 3) to the formation of solid-phase bulk $\mathrm{Pb}^{\circ}$ on top of relatively densely packed atoms of adsorbed $\mathrm{Pb}^{\circ}$ above a certain threshold coverage (horizontal dashed line).

Region B: There is adsorptive deposition under the Henry regime, so linearity holds (dark grey background). This is confirmed once more by the value of $(6.11 \pm 0.48) \times 10^{3} \mathrm{C} \mathrm{M}^{-1}$ obtained for the average $\eta_{\theta}$ (in agreement with the value mentioned in section 4.2). We can work with AGNES as usual (i.e. exploiting eqn. (9)). But, if we overcome the coverage threshold (corresponding to entering into region A), then multi-layer deposition starts to occur (runaway deposition) and equilibrium is no longer attained.

Region C: For very low gains (pale grey zone), the deposition potential is less negative and it is not able to trigger bulk deposition, so the linear regime extends beyond the threshold ( $Q<0.6 \mu \mathrm{C}$ in this experiment) because of UPD. AGNES can be applied as usual, although some bending (e.g. following Langmuir isotherm) is expected (but, so far, not seen) when saturation of the electrode surface was approached.

Figure 3 indicates that the interval of linearity between the charge and the concentration of the free $\mathrm{Pb}(\mathrm{II})$, clearly depends on the gain applied. The use of higher gains produces higher charges, but because bulk deposition phenomenon conditions are reached at $Q \sim 0.60 \mu \mathrm{C}$, the upper limit of linearity is compromised. For the highest gain, $Y=370$, it is not possible to work with concentrations of free $\mathrm{Pb}(\mathrm{II})$ above $230 \mathrm{nM}$ and the window of linearity varies from the LOD of $6 \mathrm{nM}$ to that value. When lower gains are 
applied, the upper limit of linearity increases, but because higher detection limits are attained, the lower limit of linearity also increases. For example for $Y=185$ the workable concentration range will be between 11 and $600 \mathrm{nM}$. For very low gains, such as $Y=51$, bulk deposition was not observed (in the probed concentration range) and in this case, just the lower limit of linearity is affected, corresponding to the LOD of $34 \mathrm{nM}$.

\section{Speciation results}

The knowledge of the free metal concentration in equilibrium in a system containing complex species leads to the computation of the stability constant via:

$$
K^{\prime}=\frac{C_{\mathrm{T}, \mathrm{Pb}}}{\left[\mathrm{Pb}^{2+}\right]}-1
$$

For each speciation assay and before adding the ligand polystyrene sulfonate (PSS) or iminodiacetate (IDA), a calibration experiment was performed in $\mathrm{NaNO}_{3}$ solutions (at the ionic strength and $\mathrm{pH}$ to be used in the speciation experiment) with total concentrations of $\mathrm{Pb}$ (II) ranging from $1.25 \times 10^{-7}$ to $5.0 \times 10^{-7} \mathrm{M}$. The deposition potential and, thus, the gain used in the calibration was the same as in the speciation experiments (details in sections 4.4.1 and 4.4.2). The charge obtained was then plotted as a function of the free concentration of $\mathrm{Pb}(\mathrm{II})$ computed with visual MINTEQ (Q vs $\left[\mathrm{Pb}^{2+}\right]$ ), allowing the determination of the product Y $\eta_{\theta}$ (required for eqn. (9)).

\section{$\mathrm{Pb}(\mathrm{II})-\mathrm{PSS}$ system}

AGNES-SCP experiments at $\mathrm{Bi}$ film electrode with $\mathrm{Pb}(\mathrm{II})$ in the presence of different concentrations of PSS $\left(M_{\mathrm{w}}=4 \mathrm{KDa}\right)$ were performed in $\mathrm{NaNO}_{3} 0.01 \mathrm{M}$ medium at $\mathrm{pH} 4$ and for a total concentration of $\mathrm{Pb}(\mathrm{II})$ of $5 \times 10^{-7} \mathrm{M}$. The measurements were performed using two different deposition potentials $E_{1}:-0.495 \mathrm{~V}$ for $t_{1}$ of $210 \mathrm{~s}$ and -0.505 $\mathrm{V}$ for $t_{1}$ of $240 \mathrm{~s}$. The results obtained for the experimental stability constant $K^{\prime}$ calculated 
by means of eqn. (15), were compared with those obtained by AGNES-SCP at Hg film electrodes (Table 2). The Hg film electrode (thickness of $28 \mathrm{~nm}$ ) was prepared following the procedure described by Rocha el al. ${ }^{30}$. AGNES parameters were: $E_{1}=-0.460 \mathrm{~V}$ for $t_{1}$ of 210 and $300 \mathrm{~s}$.

The $K^{\prime}$ values obtained from the free concentration of lead(II) in the $\mathrm{Pb}$ (II)-PSS system and for all the concentrations of ligand used are identical, within the experimental error, at both $\mathrm{Bi}$ and $\mathrm{Hg}$ film electrodes. Additionally, the $K^{\prime}$ values increase linearly with the concentration of PSS at $\mathrm{Bi}$ (slope $=1.17 \times 10^{-6}$ and $\left.r=0.998\right)$ and $\mathrm{Hg}$ (slope $=1.24 \times 10^{-6}$ and $r=0.997$ ) film electrodes.

Table 2. Stability constant $K^{\prime}$ and free concentration of lead(II), $\left[\mathrm{Pb}^{2+}\right]$, obtained for the interaction of $5.0 \times 10^{-7} \mathrm{M}$ of $\mathrm{Pb}$ (II) with different total concentration of PSS $\left(c_{T, \mathrm{~L}}\right)$ at both $\mathrm{Bi}$ and $\mathrm{Hg}$ film electrodes. The experiments were performed in $0.01 \mathrm{M} \mathrm{NaNO}_{3}$ at $\mathrm{pH} 4$.

\begin{tabular}{|c|cc|cc|}
\cline { 2 - 5 } \multicolumn{1}{c|}{} & \multicolumn{4}{c|}{ Pb-PSS (4KDa) } \\
\hline$C_{\mathrm{T}, \mathrm{L}}(\mathrm{M})$ & {$\left[\mathrm{Pb}^{2+}\right](\mathrm{nM})$} & $K^{\prime}$ AGNES & {$\left[\mathrm{Pb}^{2+}\right](\mathrm{nM})$} & $K^{\prime}$ AGNES \\
\hline $5.0 \times 10^{-5}$ & $63.6 \pm 1.9$ & $6.68 \pm 0.33$ & $60.0 \pm 2.6$ & $7.41 \pm 0.38$ \\
$7.5 \times 10^{-5}$ & $47.6 \pm 3.4$ & $9.54 \pm 0.72$ & $43.1 \pm 0.1$ & $10.7 \pm 0.1$ \\
$9.9 \times 10^{-5}$ & $35.3 \pm 1.3$ & $13.0 \pm 0.6$ & $34.2 \pm 1.3$ & $13.7 \pm 0.6$ \\
$2.0 \times 10^{-4}$ & $20.4 \pm 0.6$ & $23.3 \pm 0.7$ & $19.6 \pm 0.8$ & $24.8 \pm 1.1$ \\
\hline
\end{tabular}

\section{$\mathrm{Pb}(\mathrm{II})-$ IDA system}

The experiments with $\mathrm{Pb}(\mathrm{II})$ in the presence of IDA were conducted at $\mathrm{pH} 6$ and in $0.1 \mathrm{M} \mathrm{NaNO}_{3}$ media. The deposition potentials $E_{1}$ and the time $t_{1}$ needed to attain AGNES conditions were optimized (see section S4 of the supporting information). The measurements were performed using two different deposition potentials $E_{1}$ : $-0.520 \mathrm{~V}$ for $t_{1}$ of 210 and $240 \mathrm{~s}$ and $-0.525 \mathrm{~V}$ for $t_{1}$ of $240 \mathrm{~s}$ and $300 \mathrm{~s}$. Table 3 shows the values of the stability constant $K^{\prime}$ and free concentration of lead(II) obtained by AGNES-SCP at Bi film electrodes. 
Table 3. Stability constant $K^{\prime}$ and free concentration of lead(II) $\left[\mathrm{Pb}^{2+}\right]$ obtained by AGNES-SCP at $\mathrm{Bi}$ film electrode for $\mathrm{Pb}(\mathrm{II})$-IDA system and using different total concentrations of IDA $\left(c_{\mathrm{T}, \mathrm{L}}\right)$. The experiments were performed in $0.01 \mathrm{M} \mathrm{NaNO}_{3}$ at $\mathrm{pH} 6$ and for a total concentration of $\mathrm{Pb}(\mathrm{II})$ of $5.0 \times 10^{-7} \mathrm{M}$. Additionally the theoretical $K^{\prime}$ and obtained by SSCP at Bi film electrode ${ }^{28}$ are also provided.

\begin{tabular}{ccccc}
\multicolumn{5}{c}{ Pb-IDA } \\
$c_{\mathrm{T}, \mathrm{L}}(\mathrm{M})$ & {$\left[\mathrm{Pb}^{2+}\right](\mu \mathrm{M})$} & $K^{\prime}$ AGNES & $K^{\prime}$ SSCP & $K^{\prime}$ Theoretical \\
\hline $9.6 \times 10^{-5}$ & $0.188 \pm 0.035$ & $1.72 \pm 0.58$ & $1.34(1.00-1.74)$ & 1.59 \\
$1.9 \times 10^{-4}$ & $0.111 \pm 0.011$ & $3.47 \pm 0.38$ & $2.98(2.40-3.65)$ & 3.15 \\
$2.9 \times 10^{-4}$ & $0.084 \pm 0.003$ & $4.84 \pm 0.20$ & $4.70(3.46-5.09)^{*}$ & $4.7^{*}$ \\
\hline
\end{tabular}

These values obtained for $K$ ' were compared with those computed from SSCP curves at Bi film electrodes under identical experimental conditions ${ }^{28}$ and also with the theoretical values calculated using $\log \left(K / \mathrm{M}^{-1}\right)=7.45$ and a deprotonation degree $\alpha_{\mathrm{pH}}=5.88 \times 10^{-4}$ (values reported in the literature ${ }^{44}$ ). Within the experimental error, there is a good agreement between the measured values by both AGNES-SCP and SSCP techniques, but the values obtained tend to be slightly higher at Bi film electrodes.

\section{CONCLUSIONS}

The pre-plated Bi film electrode was successfully implemented in speciation analysis of the free concentration of $\mathrm{Pb}(\mathrm{II})$ in aqueous samples by AGNES. The measured charge $Q$ increases linearly with the free concentration of $\mathrm{Pb}(\mathrm{II})$ and the interval of linearity is highly dependent on the gain $Y$ applied, and it breaks at an approximate constant $Q$ value. This could be attributed to the lack of AGNES equilibrium, resulting from the formation of solid-phase bulk $\mathrm{Pb}^{\circ}$ on top of relatively close atoms of adsorbed $\mathrm{Pb}^{\circ}$ for a certain fixed coverage. The good sensitivity, stability and the lower LOD 
obtained with Bi film electrodes demonstrate its potentialities to perform speciation analysis by AGNES, making it an excellent and green alternative to the conventional mercury electrodes.

Further work is needed to extend the use of AGNES with Bi film electrodes to other metal ions, such as cadmium and zinc, understand the effect of the structure of the Bi film on the performance of the electrode and the presence of interferences (e.g. other metals, such as copper).

\section{Acknowledgments}

The authors thank MSc. Nuno G. Alves, Ana M. Rosa da Costa and José A. Moreira for the synthesis of polystyrene sulfonate polymer. This work is supported by Portuguese funding through Fundação para a Ciência e a Tecnologia (FCTANR/AAGMAA/0065/2012), Faculdade de Ciências e Tecnologia (Pest-OE/EQB/LA0023/2013 and PEst-OE/QUI/UI4023/2014) and by the Spanish Ministry of Science (projects CTM2012-39183 and CTM2013-48967). L.S. Rocha also thanks FCT project FCTANR/AAG-MAA/0065/2012 for her grant.

\section{Supporting Information for Publication}

This information is available free of charge via the Internet at http://pubs.acs.org/. The following studies were included in the supporting information: the study of the stripping regime, SSCP curves of $\mathrm{Pb}(\mathrm{II})$ obtained with $\mathrm{Bi}-\mathrm{FE}$ in $0.01 \mathrm{M} \mathrm{NaNO}_{3}$ media, the Nernstian behaviour of the charge $Q$ with the gain $Y$, the trajectories $Q$ vs $t_{1}$ and calibrations curves of $\mathrm{Pb}(\mathrm{II})$ in $0.1 \mathrm{M} \mathrm{NaNO}_{3}$ media. These studies will be a complement to the results presented in the manuscript.

\section{REFERENCES}

(1) Buffle, J. Complexation Reactions in Aquatic Systems. An Analytical Approach., Ellis Horwood Limited: Chichester, 1988. 
(2) Pesavento, M.; Alberti, G.; Biesuz, R. Anal.Chim.Acta 2009, 631, 129-41.

(3) Kalis, E. J. J.; Weng, L. P.; Temminghoff, E. J. M.; van Riemsdijk, W. H. Anal.Chem. 2007, 79, 1555-63.

(4) Chito, D.; Weng, L.; Galceran, J.; Companys, E.; Puy, J.; van Riemsdijk, W. H.; van Leeuwen, H. P. Sci.Total Envir. 2012, 421-422, 238-44.

(5) Noel, S.; Tercier-Waeber, M. L.; Lin, L.; Buffle, J.; Guenat, O.; Koudelka-Hep, M. Electroanal. 2006, 18, 2061-69.

(6) Parthasarathy, N.; Buffle, J. Anal.Chim.Acta 1994, 284, 649-59.

(7) Tomaszewski, L.; Buffle, J.; Galceran, J. Anal.Chem. 2003, 75, 893-900.

(8) Buhlmann, P.; Pretsch, E.; Bakker, E. Chem.Rev. 1998, 98, 1593-687.

(9) Serrano, N.; Díaz-Cruz, J. M.; Ariño, C.; Esteban, M.; Puy, J.; Companys, E.; Galceran, J.; Cecilia, J. J.Electroanal.Chem. 2007, 600, 275-84.

(10) Galceran, J.; Companys, E.; Puy, J.; Cecília, J.; Garcés, J. L. J.Electroanal.Chem. 2004, 566, 95-109.

(11) Companys, E.; Puy, J.; Galceran, J. Environ.Chem. 2007, 4, 347-54.

(12) Galceran, J.; Huidobro, C.; Companys, E.; Alberti, G. Talanta 2007, 71, 1795-803.

(13) Zavarise, F.; Companys, E.; Galceran, J.; Alberti, G.; Profumo, A. Anal.Bioanal.Chem. 2010, 397, 389-94.

(14) Diaz-de-Alba, M.; Galindo-Riano, M. D.; Pinheiro, J. P. Environ.Chem. 2014, 11, 137-49.

(15) Galceran, J.; Lao, M.; David, C.; Companys, E.; Rey-Castro, C.; Salvador, J.; Puy, J. J.Electroanal.Chem. 2014, 722-723, 110-18.

(16) Parat, C.; Authier, L.; Aguilar, D.; Companys, E.; Puy, J.; Galceran, J.; Potin-Gautier, M. Analyst. 2011, 136, 4337-43.

(17) Domingos, R. F.; Huidobro, C.; Companys, E.; Galceran, J.; Puy, J.; Pinheiro, J. P. J.Electroanal.Chem. 2008, 617, 141-48.

(18) Huidobro, C.; Companys, E.; Puy, J.; Galceran, J.; Pinheiro, J. P. J.Electroanal.Chem. 2007, 606, 134-40.

(19) Rocha, L. S.; Companys, E.; Galceran, J.; Carapuca, H. M.; Pinheiro, J. P. Talanta 2010, $80,1881-87$.

(20) Aguilar, D.; Galceran, J.; Companys, E.; Puy, J.; Parat, C.; Authier, L.; Potin-Gautier, M. Phys.Chem.Chem.Phys. 2013, 15, 17510-21.

(21) Parat, C.; Aguilar, D.; Authier, L.; Potin-Gautier, M.; Companys, E.; Puy, J.; Galceran, J. Electroanal. 2011, 23, 619-27. 
(22) Parat, C., Authier, L., Castetbon, A., Aguilar, D., Companys, E., Puy, J., Galceran, J., and Potin-Gautier, M. Free $\mathrm{Zn2}+$ determination in natural freshwater of the Pyrenees: towards on-site measurements with AGNES. Environmental Chemistry . 2014.

Ref Type: In Press

(23) Economou, A. Trac-Trend Anal Chem 2005, 24, 334-40.

(24) Serrano, N.; Alberich, A.; Diaz-Cruz, J. M.; Arino, C.; Esteban, M. Trac-Trend Anal Chem 2013, 46, 15-29.

(25) Svancara, I.; Prior, C.; Hocevar, S. B.; Wang, J. Electroanal. 2010, 22, 1405-20.

(26) Wang, J. Electroanal. 2005, 17, 1341-46.

(27) Rocha, L. S.; Pereira, E.; Duarte, A. C.; Pinheiro, J. P. Electroanal. 2011, 23, 1891-900.

(28) Pinheiro, J. P.; Rocha, L. S.; Goveia, D.; Town, R. M. Environ.Chem. 2014, 11, 150-57.

(29) Barsbay, M.; Guven, O.; Davis, T. P.; Barner-Kowollik, C.; Barner, L. Polymer 2009, 50, 973-82.

(30) Rocha, L. S., Botero, W. G., Alves, N., Moreira, J., Costa, A. M. R., and Pinheiro, J. P. Ligand size polydispersity effect on SSCP signal. Electrochimica Acta . 2015.

Ref Type: In Press

(31) Monterroso, S. C. C.; Carapuca, H. M.; Duarte, A. C. Electroanal. 2003, 15, 1878-83.

(32) Kong, D. D.; Chen, Y. M.; Wan, P. Y.; Liu, S. Y.; Khan, Z. U.; Men, B. Electrochim.Acta. 2014, 125, 573-79.

(33) Bard, A. J.; Faulkner, L. R. Electrochemical Methods. Fundamentals and Applications., Second edition ed.; John Wiley \& Sons, Inc.: New York, 2001.

(34) Taguchi, S.; Kondo, M.; Mori, H.; Aramata, A. Electrochim.Acta. 2013, 111, 642-55.

(35) Galceran, J.; Chito, D.; Martinez-Micaelo, N.; Companys, E.; David, C.; Puy, J. J.Electroanal.Chem. 2010, 638, 131-42.

(36) Herzog, G.; Arrigan, D. W. M. Trac-Trend Anal Chem 2005, 24, 208-17.

(37) Kirowa-Eisner, E.; Bonfil, Y.; Tzur, D.; Gileadi, E. J.Electroanal.Chem. 2003, 552, 171-83.

(38) Hamelin, A.; Lipkowski, J. J.Electroanal.Chem. 1984, 171, 317-30.

(39) Vicente, V. A.; Bruckenstein, S. Anal.Chem. 1973, 45, 2036-43.

(40) Motheo, A. J.; Gonzalez, E. R.; Tremilliosi, G.; Rakotondrainibe, A.; Leger, J. M.; Beden, B.; Lamy, C. J.Brazil.Chem.Soc. 1998, 9, 31-38.

(41) Brand, M.; Eshkenazi, I.; Kirowa-Eisner, E. Anal.Chem. 1997, 69, 4660-64.

(42) Bort, H.; Juttner, K.; Lorenz, W. J.; Schmidt, E. J.Electroanal.Chem. 1978, 90, 413-24.

(43) Szabo, S. Int.Rev.Phys.Chem. 1991, 10, 207-48. 
(44) Anderegg, G. Helv.Chim.Acta 1964, 47, 1801-\&.

\section{For TOC only}

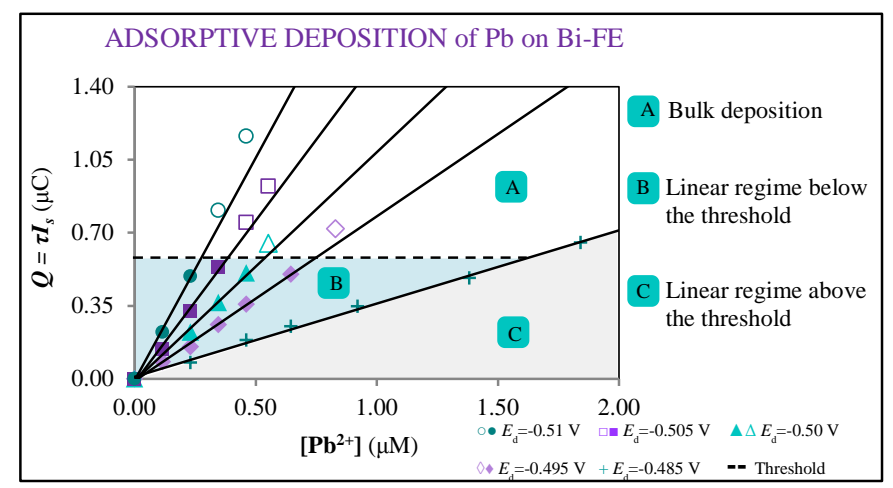

